\title{
Clinical Outcomes of Secondary Scleral-Sutured Foldable Hydrophilic Acrylic Intraocular Lens Placement by Trainees: A Single-Site Analysis
}

This article was published in the following Dove Press journal: Clinical Ophthalmology

\author{
Yesha S Shah (D' \\ Angela Y Zhu' \\ Sidra I Zafar' \\ Daniel Sarezky' \\ Ximin $\mathrm{Li}^{2}$ \\ TY Alvin Liu' \\ Mira M Sachdeva' \\ Fasika A Woreta (D) \\ 'Department of Ophthalmology, Wilmer \\ Eye Institute, Johns Hopkins Medical \\ Institutions, Baltimore, MD, USA; \\ ${ }^{2}$ Department of Biostatistics, Johns \\ Hopkins Bloomberg School of Public \\ Health, Baltimore, MD, USA
}

Purpose: To evaluate the outcomes of a 4-point scleral-fixated foldable Akreos AO60 intraocular lens (IOL) insertion using Gore-Tex suture performed by trainees under supervision of a single attending surgeon.

Methods: Retrospective chart review for 53 eyes of 50 patients whose surgery was performed by trainees under supervision of a single surgeon between 2015 and 2018 at a tertiary care hospital (Johns Hopkins Wilmer Eye Institute, Baltimore, MD). Indications for surgery, preoperative risk factors, and intraoperative techniques were analyzed. Outcome measures included final best-corrected visual acuity (BCVA), change in BCVA, difference between expected and final spherical equivalent (SE), and postoperative complications.

Results: Mean patient age was 62.8 years (range 26.9 to 88.4 ). The most common indication for surgery was IOL dislocation (59.6\%) due to trauma in 21 cases $(40.4 \%)$ and pseudoexfoliation in $6(11.5 \%)$. Combined pars plana vitrectomy was performed simultaneously in 46 cases $(88.5 \%)$. Mean BCVA improved from $20 / 100$ to $20 / 40(p<0.001)$. The difference between expected and final SE was within $1.0 \mathrm{D}$ in 28 cases $(53.8 \%)$. Postoperative hypotony occurred in 12 eyes $(21.2 \%)$ on day 1 ; all were resolved at last follow-up. Postoperative cystoid macular edema (CME) occurred in 20 cases $(38.5 \%)$; $11(21.2 \%)$ persisted through last follow-up.

Conclusion: Scleral-fixation of Akreos AO60 IOL in absence of capsular support can be performed by trainees under supervision and results in effective visual rehabilitation. Postoperative CME occurred at a higher rate than previously reported in the literature. Future studies should assess the rates of postoperative complications amongst different techniques of secondary IOL fixation performed by trainees to determine which is the safest. Keywords: secondary IOL, scleral-fixated IOL, scleral-sutured IOL, Akreos, clinical outcomes

\section{Introduction}

In the absence of adequate capsular support for standard intraocular lens (IOL) placement during cataract surgery, there are a variety of techniques for secondary IOL fixation. A posterior chamber IOL (PCIOL) can be placed in the ciliary sulcus without additional support if the anterior capsular rim remains intact. ${ }^{1}$ If anterior capsular support is lost, alternative approaches include anterior chamber angle support, fixation to the iris with sutures or iris claw, and fixation to the sclera with or without sutures., ${ }^{2,3}$

These alternative options for IOL placement in the absence of adequate capsular support all have numerous individual risks for complications as well as collective
Correspondence: Fasika A Woreta Department of Ophthalmology, Wilmer Eye Institute, Johns Hopkins Medical Institutions, $600 \mathrm{~N}$. Wolfe St., Wilmer B-29, Baltimore, MD, 2I 287, USA

Tel + | 410 955-8265

Fax + 410 614-9632

Email fworetal@jhmi.edu 
disadvantages. ${ }^{1,2,4-8}$ A recent ophthalmic technologic assessment by the American Academy of Ophthalmology ${ }^{9}$ found no evidence of superiority of any one method of fixation over another as they all have similar visual acuity outcomes and safety profiles. ${ }^{4,9,10}$ However, anterior chamber IOLs (ACIOLs) should be avoided in patients with iris abnormalities, shallow anterior chambers, or if future corneal transplantation may be necessary. ${ }^{9}$ Additionally, iris-fixated IOLs may not be possible in the presence of significant iris trauma and may re-sublux over time. ${ }^{11}$ Scleral fixation is a suitable alternative and can be accomplished through a variety of techniques including suturing or sutureless intrascleral haptic fixation. ${ }^{2}$

Techniques for secondary IOL fixation are associated with a learning curve and may be challenging to teach surgeons-intraining and novice surgeons. In recent years, the hydrophilic acrylic Akreos AO60 lens (Bausch \& Lomb, Rochester, New York) has been co-opted for scleral suturing techniques, due to its four looped haptics allowing for four-point scleral fixation, which theoretically reduce risks of lens tilt and decentration. ${ }^{6}$ While the initial technique was first described in 2010, the current standard protocol involves off-label use of the CV8 Gore-Tex polytetrafluoroethylene monofilament suture for scleral fixation of IOL haptics, with preliminary studies confirming its safety profile. ${ }^{11-13}$ Multiple case series have reported overall positive clinical outcomes in conjunction with concurrent pars plana vitrectomy, with minimal complication rates and comparable visual outcomes to those of ACIOL implantation with up to 3 years of follow-up. ${ }^{14-19}$

While these studies have shown acceptable outcomes of Gore-Tex scleral-sutured Akreos AO60 lenses as performed by expert surgeons, ${ }^{20}$ to our knowledge, there are no published reports of whether this technique is equally safe and effective when performed by novice surgeons, including an assessment of surgical complications and refractive outcomes. Since the technical challenges of learning a new technique often present a significant barrier to widespread adoption, the main purpose of our study was to evaluate clinical outcomes of secondary scleral-sutured Akreos AO60 lenses performed by novice surgeons-intraining under supervision of a single attending surgeon.

\section{Patients and Methods}

\section{Patient Cases}

A retrospective chart review was performed of all patients who underwent scleral fixation of an Akreos AO60 (Bausch and Lomb, Bridgewater, NJ, USA) IOL using CV-8 (USP 7-0) Gore-Tex suture (W.L. Gore \&
Associates, Newark, DE, USA) by a PGY4 resident or fellow under supervision of a single attending (F.A.W.) at Johns Hopkins Wilmer Eye Institute from November 1, 2015 (initial adoption of technique) to August 31, 2018. This study was carried out in adherence to the Declaration of Helsinki and was approved by the Institutional Review Board of our institute in which requirement for informed consent was waived due to the retrospective nature of the study. All work was compliant with the Health Insurance Portability and Accountability Act (HIPAA). All patients were identified from surgical operative reports for current procedural terminology (CPT) codes 66,985 (insertion of IOL prosthesis, secondary implant, not associated with concurrent cataract removal), 66,682 (suture to the iris or ciliary body during cataract removal), 66,986 (exchange of IOL), and 66,825 (repositioning of an IOL prosthesis).

Patient records meeting the above criteria were reviewed, and the following baseline preoperative data were collected: age at time of surgery, gender, surgery date, eye laterality, reason for loss of capsular support (ie surgical complication, trauma, pseudoexfoliation, Marfan syndrome), best-corrected visual acuity (BCVA), manifest refraction, keratometry, and axial length. Conventional biometry was used for IOL calculations and expected postoperative spherical equivalent (SE) was calculated using the Sanders-Retzlaff-Kraff theoretical (SRK/T) formula assuming sulcus IOL location. If trauma or other anatomic factors precluded this, ultrasound biometry utilizing keratometry values of the fellow eye was used for IOL power calculations.

Intraoperative data collected included: IOL power implanted, sclerotomy location (measured in $\mathrm{mm}$ behind limbus), whether combined with pars plana vitrectomy (PPV) and gauge of trocar used to make sclerotomy incisions, and intraoperative complications. Postoperative BCVA on all time points available, which typically included day 1 , week 1 , month 1 , month 3 , month 6 , and one year, as well as BCVA at last follow-up, were collected. Other postoperative data collected included: intraocular pressure (IOP) at day 1, week 1, month 1, and last follow-up, duration of follow-up period, and manifest refraction. All postoperative complications were recorded. Hypotony was defined as a new onset of an IOP of $\leq 6 \mathrm{~mm}$ $\mathrm{Hg}$ at postoperative day 1 , week 1 , month 1 , or last-follow -up, which was based on the World Glaucoma Association guidelines. ${ }^{21}$ Cystoid macular edema was defined as newonset postoperative macular edema that was confirmed with spectral domain optical coherence tomography 
(OCT). OCT imaging of the macula was routinely performed at the postoperative month 1 visit in all cases and at other time points when clinically indicated. Corneal edema was defined as new-onset postoperative edema that persisted $>1$ month.

Snellen visual acuities were converted to logarithm of the minimum angle of resolution ( $\log \mathrm{MAR})$ equivalents. The primary outcome measures were best postoperative BCVA, change in BCVA, difference between expected and final spherical equivalent (SE), and postoperative complications. Using Stata software (StataCorp LLC, College Station, TX, USA) and R 3.5.0 (R Core Team, Vienna, Austria) to compute paired Student's $t$ test analysis or Fisher's exact test, a $p$-value $<0.05$ was considered statistically significant.

\section{General Surgical Technique}

The same anterior segment attending surgeon (F.A.W.) supervised all procedures performed by a PGY4 resident or fellow using a standard ab externo scleral fixation technique with $\mathrm{CV}-8$ Gore-Tex suture, which has been described separately. ${ }^{13}$ In each case, the resident performed $75 \%$ or more of the surgery with verbal instructions or intervention by the attending when necessary. Briefly, three-clock-hour conjunctival peritomies are created 180 degrees apart, centered at 3 and 9 o'clock. Four separate sclerotomies are made (two on each side, $4 \mathrm{~mm}$ apart centered at 3 and 9 o'clock) with each sclerotomy the same designated distance $(2-3.5 \mathrm{~mm})$ from the limbus, using either 23-gauge or 25-gauge equivalent microvitreoretinal (MVR) blade or trocar. A $1.0 \mathrm{~mm}$ anterior chamber paracentesis and $4.5 \mathrm{~mm}$ corneal incision are made using a keratome, and ophthalmic viscosurgical device (OVD) is instilled into the anterior chamber. Each half of the suture is looped through each pair of eyelets of the IOL. Each end of the suture is then passed into the anterior chamber through the main incision and externalized through one of the four sclerotomy sites. The IOL is then inserted into the anterior chamber, and the sutures are tied and the IOL is centered. In all cases, the knots are trimmed and rotated into a sclerotomy site. If a sclerotomy is noted to be leaking, a separate 8-0 Vicryl suture is used to suture the sclerotomy incision. The conjunctival peritomies are then closed at the limbus. All patients either had prior posterior vitrectomy or had it concurrently at the same time of secondary IOL fixation. The PPV was performed by a separate vitreoretinal fellow or attending. In patients with a prior vitrectomy, a Lewicky infusion was placed to maintain the anterior chamber and avoid intraoperative hypotony.

\section{Postoperative Management}

Patients were treated with a postoperative medical regimen including a topical antibiotic eyedrop for 1 week, as well as a topical steroid and nonsteroidal anti-inflammatory drug (NSAID) for at least 1 month (exact duration and taper determined on a case-by-case basis).

\section{Results}

\section{Baseline Patient Demographics}

Baseline characteristics are detailed in Table 1. A total of 52 eyes of 49 patients (27 right eyes and 25 left eyes) were included. Fourteen PGY4s and 9 fellows performed the surgeries in this cohort. Forty-three cases were performed by PGY4 residents and 9 cases were performed by fellows. The mean age at time of surgery in our patient cohort was 62.8 years (standard deviation 12.2 years, median 62.7 years, range 26.9 to 88.4 years, ), and 31 patients $(59.6 \%)$ were male. Mean follow-up length was 11.1 months (standard deviation 7.5 months, median 10.5 months, range 1 to 25 months), with 5 patients (9.6\%) having less than 3 months of follow-up. Mean preoperative logMAR BCVA was $0.71 \pm 0.65(20 / 101$ Snellen equivalent), with 10 eyes (19.2\%) having $\geq 20 / 40$ Snellen BCVA and 38 eyes (73.1\%) with $\geq 20 / 200$ Snellen BCVA.

Table 1 describes indications for scleral-fixated IOL. In eyes with dislocated IOLs, repositioning was considered at first, but ultimately clinical assessment and judgment deemed a secondary IOL placement the preferred option due to complete loss of capsular support and severity of dislocation. Forty-six eyes (88.5\%) underwent concurrent PPV with a vitreoretinal surgeon while the other 6 eyes (11.5\%) had a prior PPV. The primary associated risk factors including globe trauma, pseudoexfoliation, and Marfan syndrome are shown in Table 2. Two cases required subsequent corneal transplant (1 Descemet stripping endothelial keratoplasty, 1 penetrating keratoplasty), due to pre-existing corneal edema and trauma.

Intraoperative characteristics were also analyzed. Cases that were performed earlier in our cohort had sclerotomies that were $2 \mathrm{~mm}$ posterior to the limbus, but the technique was refined, and later cases only used sclerotomies $3.25-3.5 \mathrm{~mm}$ posterior to the limbus. In eyes that underwent a concurrent PPV, either a 23-gauge or 25-gauge needle was used to create the sclerotomy, 
Table I Cohort Baseline Characteristics, Indications for Scleral-Fixated IOL, and Risk Factors of All Cases Reviewed

\begin{tabular}{|c|c|c|}
\hline & n (\%) & Mean \pm SD \\
\hline \multicolumn{3}{|l|}{ Demographics } \\
\hline Age at time of surgery (years) & 52 & $\begin{array}{c}62.8 \pm 12.2(\text { range } 26.9-88.4 \\
\text { median } 62.7)\end{array}$ \\
\hline Follow-up duration (months) & 52 & $\mathrm{II} . \mathrm{I} \pm 7.5$ \\
\hline Male sex & $31(59.6 \%)$ & \\
\hline \multicolumn{3}{|c|}{ Primary indication for scleral-fixated IOL } \\
\hline Aphakia & $9(17.3 \%)$ & \\
\hline Subluxed native lens & $8(15.4 \%)$ & \\
\hline Dislocated IOL & 31 (59.6\%) & \\
\hline Complicated cataract surgery & $4(7.7 \%)$ & \\
\hline \multicolumn{3}{|l|}{ Associated risk factors } \\
\hline Globe trauma & $21(40.4 \%)$ & \\
\hline Pseudoexfoliation (PXF) & $6(11.5 \%)$ & \\
\hline Combined pars plana vitrectomy (PPV) & $46(88.5 \%)$ & \\
\hline Marfan syndrome & $2(3.8 \%)$ & \\
\hline
\end{tabular}

Abbreviations: $n$, number; SD, standard deviation; IOL, intraocular lens.

decided based on surgeon preference. The 6 eyes with a prior PPV underwent sclerotomy with a 25 -gauge needle. Out of the 46 eyes that underwent concurrent PPV, 5 eyes $(10.9 \%)$ had a posterior vitreous detachment induction. All cases underwent base shaving at the sclerotomy positions.

\section{Postoperative Outcomes}

Mean $\log$ MAR of best postoperative BCVA at any time point was $0.26 \pm 0.26$ (20/36 Snellen equivalent), and the mean change in logMAR BCVA was $-0.45 \pm 0.60$, which was significantly improved from preoperative mean logMAR BCVA $(P<0.001$, Table 2$)$. There were 37 cases $(71.2 \%)$ who achieved $\geq 20 / 40$ Snellen BCVA postoperatively, and 50 cases $(96.2 \%)$ with $\geq 20 / 200$ Snellen BCVA. These cutoffs were chosen based on the definitions of low vision and legal blindness, respectively. ${ }^{22}$ Eleven eyes $(21.2 \%)$ had unchanged or reduced BCVA at last follow-up visit; of those, four $(7.7 \%)$ had greater than $0.1 \log$ MAR reduction in BCVA (lost more than 1 Snellen line equivalent) due to persistent CME (2 cases), central retinal vein occlusion (CRVO, 1 case), and corneal edema (1 case).
Postoperative refractive data was available for 48 cases (92.3\%). With sclerotomies $3.25-3.5 \mathrm{~mm}$ posterior to the limbus, in the majority of patients, distance vision was preferred so the target was plano. With sclerotomies $2 \mathrm{~mm}$ posterior to the limbus, the target was plano with a sulcus adjustment. The mean difference between expected and postoperative SE was $-0.87 \mathrm{D}$. Twenty-eight cases (53.8\%) resulted in a postoperative $\mathrm{SE}$ within $\pm 1.0 \mathrm{D}$ of the calculated target SE. However, a significant majority $(\mathrm{n}=40,76.9 \%, P<$ 0.001 ) had a myopic shift between expected and actual postoperative SE; 18 of those eyes (34.6\%) had more than -1.0 $\mathrm{D}$ difference between target and actual SE (Figure 1).

\section{Surgical Complications}

The list of all intraoperative and postoperative complications is detailed in Table 2. There were 3 eyes $(5.8 \%)$ with lens decentration at postoperative week 1 . In 3 cases $(5.8 \%)$, intraoperative retinal tears were found and treated with laser retinopexy. Postoperative CME was noted in 20 eyes (38.5\%), with 11 (21.2\%) persistent at last follow-up. Postoperative hypotony occurred in 11 eyes $(21.2 \%)$, but only $2(3.8 \%)$ were persistent through postoperative week 1 , and only 1 was persistent 
Table 2 Postoperative Outcomes of All Cases Reviewed. Patient with Hand Motions or Worse Vision Were Excluded from Conversion to LogMAR BCVA

\begin{tabular}{|c|c|c|}
\hline & n (\%) & Mean \pm SD \\
\hline Postoperative BCVA & & $0.26 \pm 0.26(\log M A R)$ \\
\hline Snellen $>20 / 200$ & $50(96.2 \%)$ & 20/36 (Snellen) \\
\hline Change in BCVA & & $-0.44 \pm 0.59(\log M A R)^{a}$ \\
\hline \multicolumn{3}{|c|}{ Postoperative refractive outcomes } \\
\hline Myopic SE & $36(69.2 \%)^{b}$ & \\
\hline Hyperopic SE & $10(19.2 \%)$ & \\
\hline \multicolumn{3}{|l|}{ Postoperative complications } \\
\hline Cystoid macular edema (CME) & $20(38.5 \%)$ & \\
\hline Resolved & $9(17.3 \%)$ & \\
\hline Persistent at last follow-up & II (2I.2\%) & \\
\hline Postoperative hypotony & $\mathrm{II}(2 \mathrm{I} .2 \%)$ & \\
\hline On PODI & $10(19.2 \%)$ & \\
\hline On POWI & $2(3.8 \%)$ & \\
\hline Ocular hypertension & $2(3.8 \%)$ & \\
\hline Corneal edema & $3(5.8 \%)$ & \\
\hline Macular hole & $\mathrm{I}(\mathrm{I} .9 \%)$ & \\
\hline IOL dislocation & $\mathrm{I}(1.9 \%)$ & \\
\hline Vitreous hemorrhage & I (I.9\%) & \\
\hline Wound leak & $\mathrm{I}(\mathrm{I} .9 \%)$ & \\
\hline Conjunctival cyst & I (I.9\%) & \\
\hline
\end{tabular}

Notes: ${ }^{\text {a Two-tailed }} p<0.001$ comparing mean logMAR postoperative to preoperative BCVA. ${ }^{\text {'Two-tailed }} p<0.001$ comparing postoperative myopic to hyperopic spherical equivalent.

Abbreviations: $n$, number; SD, standard deviation; IOL, intraocular lens; BCVA, best-corrected visual acuity; logMAR, logarithm of the minimum angle of resolution; SE, spherical equivalent; PODI, postoperative day I; POWI, postoperative week I.

through postoperative month $1(1.9 \%)$, with all cases being resolved by last follow-up. Rates of corneal edema, ocular hypertension, full-thickness macular hole, wound leak, vitreous hemorrhage, and conjunctival cyst are described in Table 2. Two cases needed epithelial debridement, 1 required repair of wound leak, 1 underwent macular hole repair, 1 needed glaucoma drainage implant surgery due to persistently elevated IOP after surgery, and 1 underwent excision of conjunctival cyst (possible suture granuloma). There were no cases of IOL opacification, suprachoroidal hemorrhage, postoperative endophthalmitis, IOL dislocation, or retinal detachment in the follow-up period.

\section{Discussion}

Various techniques for secondary IOL fixation in the absence of capsular support exist, with no evidence of superiority in terms of clinical outcomes or safety profiles for any one method. ${ }^{4,9,10}$ Despite the relative equal outcomes of all methods, secondary IOL fixation is technically challenging and there is a learning curve associated, especially for surgeons-in-training and novice surgeons.

Because no single method has been shown to be clinically superior, it is important to assess which method is the safest in the hands of surgeons-in-training and novice surgeons and easiest to teach safely. While the safety profile of Gore-Tex scleral-sutured Akreos AO60 lenses has been 


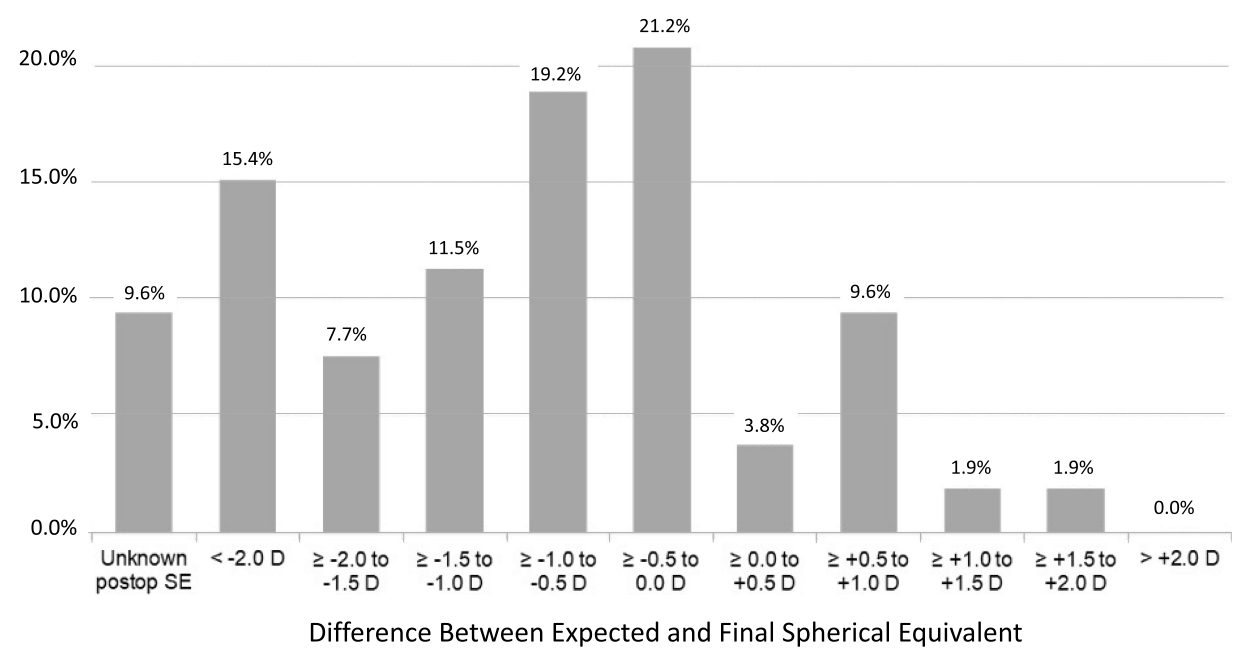

Figure I Frequency distribution of the percentage of cases having postoperative refractive outcomes within the stated range of difference between predicted target and actual postoperative spherical equivalent.

Abbreviation: D, diopter.

established in literature, no published studies have examined yet whether this procedure is effective when performed by novice surgeons. Our analysis demonstrated that this described method for secondary Akreos AO60 IOL insertion can be executed by surgeons-in-training under adequate supervision with favorable outcomes. A significant majority of patients in our study had improved BCVA postoperatively, and there were no intraoperative complications that resulted in decreased vision. To our knowledge, this study is one of the first to evaluate trainees' surgical outcomes with secondary IOL fixation and our cohort size is similar to some of the larger retrospective series of secondary scleralsutured IOL outcomes.

Many studies evaluating scleral fixation have shown a mild myopic shift from targeted postoperative refraction. ${ }^{17,20,23,24}$ Ohr et al specifically studied scleral fixation of Akreos lenses and has shown reliable postoperative refractive outcomes with a myopic shift. ${ }^{20}$ Our evaluation corroborated the results of previous studies of Gore-Tex sutured lenses with a significant majority (75\%) of our patients having a myopic shift in the difference between actual and targeted SE. Only $35 \%$ of our patients had greater than $-1.0 \mathrm{D}$ difference between predicted and actual SE with the mean difference being $-0.94 \mathrm{D}$. This is similar to a recent study that found a mean postoperative SE difference of $-0.99 \mathrm{D}$ for Gore-Tex scleral-sutured secondary IOLs. ${ }^{23}$ Our biometry calculations were performed using the SRK/T formula, which Ohr et al also used and reported similar results. ${ }^{20}$ We chose to use the SRK/T formula because Ohr et al used the same formula and we could therefore directly compare our refractive outcomes to that of an established cohort. ${ }^{20}$
Furthermore, a recent investigation demonstrated noninferiority of 4 different common IOL calculation formulas (SRK/T, Barrett II, Holladay 2, Hoffer Q) in predicting postoperative SE of scleral-fixated IOL implantation. ${ }^{24}$ The replication of previous studies' results in our cohort further shows that the scleral-fixated technique for Akreos lenses is relatively effective in the hands of surgeons-in-training and novice surgeons.

Our study also characterized rates of postoperative complications of eyes with Gore-Tex scleral-sutured Akreos AO60 lenses. Our cohort's rates of corneal edema, ocular hypertension, hypotony, vitreous hemorrhage, wound leak, and reoperations for wound leak, epithelial debridement, and tube shunt implantation for elevated IOP were very similar or lower than previously reported in the literature. ${ }^{14,16-18,25}$ To our knowledge, there have not been previous reports of the development of a full thickness macular hole or conjunctival cyst with this technique, but these complications are likely due to the increased complexity of patients at a tertiary care center and the relatively small sample sizes in previously published studies evaluating postoperative complications with Gore-Tex scleral suture Akreos AO60 lenses. Future studies should characterize the rates of postoperative complications in various techniques of scleral fixated IOLs performed by trainees to determine which has the lowest rate.

Our cohort did show a higher rate of postoperative CME (21\% persistent to last follow-up visit) compared to other studies (less than $7 \%$ ), ${ }^{11,12,16,18}$ but this is likely due to our postoperative protocol to routinely obtain OCT macula imaging at the one month follow-up period for all patients which was not obtained in prior studies. Another 
reason for the high reported rate of CME in our study may be because of the increased complexity of our cases as this study only included patients from a tertiary care center. Additionally, about $40 \%$ of our cases were secondary to globe trauma, which has previously been described in association with $\mathrm{CME}{ }^{26,27}$ Given the high rate of CME, further studies need to assess risk factors for this complication and compare rates of CME in other techniques of scleral fixated IOLs performed by trainees. All patients in our study did not get a preoperative macula OCT, which should also be considered as a baseline given the high-rate of OCT-evidence of CME in our study.

While our study upheld the efficacy of the scleralfixated technique for Akreos AO60 IOL with novice surgeons, it is important to note that it has an increased risk of opacifications due to its hydrophilicity. ${ }^{28,29}$ This may affect the surgeon's choice to use this technique, especially if there is a concomitant procedure being performed or the patient is at a high risk of retinal detachment in the future. Other commonly used techniques are the Yamane sutureless technique with CT Lucia lens and the scleral sutured technique with the enVista MX60 (Bausch \& Lomb) IOL. The enVista MX60 lens is hydrophobic and not as susceptible to opacification, which may be useful in patients with or at an increased risk of retinal detachment. ${ }^{30,31}$

However, both the sutureless Yamane technique and lens insertion and fixation of the enVista MX60 IOL are known to be technically difficult and therefore may not be the first choice in a trainee-performed procedure. ${ }^{31,32}$ Additionally, the Yamane technique is known to cause postoperative complications such as lens decentration and tilt. Due to the four eyelets on the Akreos AO60 IOL, it is relatively easy to center the lens during insertion and there is a lower risk of lens tilt compared to other techniques. ${ }^{6}$ Future studies should assess and compare the safety profiles and ease of teaching of all of these techniques to best determine which is safest in the hands of novice surgeons.

As a single-site, retrospective study, our results were primarily limited by this study design and number of cases. For attempted ascertainment of predictive factors associated with improved refractive outcomes, the low sample size resulted in inadequate statistical power for most regression analyses. There was also a moderate attrition rate with $9.6 \%$ being lost to follow-up prior to 3 months postoperatively that may have biased postoperative outcomes since longer-term follow-up data was not available for all cases. Conducted at a tertiary care eye trauma center, our study results may be subject to referral bias.
However, the fact that our results reflected data for a single primary surgeon at one institution allowed for control of many surgeon- and institution-dependent factors (eg patient selection, preoperative measurements, intraoperative technique, postoperative management) that often confound larger clinical outcomes studies.

\section{Conclusions}

Overall, our report demonstrates that Gore-Tex suture scleral fixation of Akreos AO60 IOL is an effective technique for visual rehabilitation when performed by surgeons-in-training under adequate supervision. In the future, rates of postoperative complications should be compared between various techniques of scleral fixated IOLs performed by trainees. A large retrospective multicenter study, the use of a clinical registry, or the addition of a comparison group will be useful in assessing clinical outcomes and safety profiles of a variety of secondary fixation of IOL techniques for surgeons-in-training or novice surgeons to determine which should be used in a trainee performed procedure.

\section{Funding}

There is no funding to report.

\section{Disclosure}

Dr Mira M Sachdeva reports being an ad hoc consultant/ advisory board member for Allergan, Inc, outside the submitted work. The authors reported no other potential conflicts of interest for this work.

\section{References}

1. Por YM, Lavin MJ. Techniques of intraocular lens suspension in the absence of capsular/zonular support. Surv Ophthalmol. 2005;50 (5):429-462. doi:10.1016/j.survophthal.2005.06.010

2. Dajee KP, Abbey AM, Williams GA. Management of dislocated intraocular lenses in eyes with insufficient capsular support. Curr Opin Ophthalmol. 2016;27(3):191-195. doi:10.1097/ICU.00000000 00000260

3. Dick HB, Augustin AJ. Lens implant selection with absence of capsular support. Curr Opin Ophthalmol. 2001;12(1):47-57. doi:10.1097/ 00055735-200102000-00009

4. Wagoner MD, Cox TA, Ariyasu RG, Jacobs DS, Karp CL. Intraocular lens implantation in the absence of capsular support: a report by the American academy of ophthalmology. Ophthalmology. 2003;110 (4):840-859. doi:10.1016/S0161-6420(02)02000-6

5. Davies EC, Pineda R. Complications of scleral-fixated intraocular lenses. Semin Ophthalmol. 2018;33(1):23-28. doi:10.1080/0882053 8.2017.1353808

6. Stem MS, Todorich B, Woodward MA, Hsu J, Wolfe JD. Scleralfixated intraocular lenses: past and present. $J$ Vitreoretin Dis. 2017;1 (2):144-152. doi:10.1177/2474126417690650 
7. Sindal MD, Nakhwa CP, Sengupta S. Comparison of sutured versus sutureless scleral-fixated intraocular lenses. J Cataract Refract Surg. 2016;42(1):27-34. doi:10.1016/j.jcrs.2015.09.019

8. Nudleman E, Yonekawa Y, Prenner JL. Sutureless transscleral fixation of secondary intraocular lenses. Curr Opin Ophthalmol. 2018;29 (3):210-216. doi:10.1097/ICU.0000000000000474

9. Shen JF, Deng S, Hammersmith KM, et al. Intraocular Lens Implantation in the absence of Zonular support: an outcomes and safety update: a report by the American academy of ophthalmology. Ophthalmology. 2020;127(9):1234-1258. doi:10.1016/j. ophtha.2020.03.005

10. Donaldson KE, Gorscak JJ, Budenz DL, Feuer WJ, Benz MS, Forster RKM. Anterior chamber and sutured posterior chamber intraocular lenses in eyes with poor capsular support. J Cataract Refract Surg. 2005;31(5):903-909. doi:10.1016/j.jcrs.2004.10.061

11. Fass ON, Herman WK. Four-point suture scleral fixation of a hydrophilic acrylic IOL in aphakic eyes with insufficient capsule support. J Cataract Refract Surg. 2010;36:991-996. doi:10.1016/j. jcrs.2009.12.043

12. Khan MA, Gupta OP, Smith RG, et al. Scleral fixation of intraocular lenses using Gore-Tex suture: clinical outcomes and safety profile. $\mathrm{Br}$ $J$ Ophthalmol. 2016;100(5):638-643. doi:10.1136/bjophthalmol2015-306839

13. Khan MA, Gerstenblith AT, Dollin ML, Gupta OP, Spirn MJ. Scleral fixation of posterior chamber intraocular lenses using gore-tex suture with concurrent 23-gauge pars plana vitrectomy. Retina. 2014;34 (7):1477-1480. doi:10.1097/IAE.0000000000000233

14. Terveen DC, Fram NR, Ayres B, Berdahl JP. Small-incision 4-point scleral suture fixation of a foldable hydrophilic acrylic intraocular lens in the absence of capsule support Presented at the ASCRS Symposium on Cataract, IOL and Refractive Surgery, San Diego, California, USA, April 2015. J Cataract Refract Surg. 2016;42 (2):211-216. doi:10.1016/j.jcrs.2015.10.068

15. Suture LUG. Combined 27-Gauge Pars Plana Vitrectomy and Scleral Fixation of an Akreos AO60 intraocular; 2014:1602-1604.

16. Khan MA, Samara WA, Gerstenblith AT, et al. Combined pars plana vitrectomy and scleral fixation of an intraocular lens using gore-tex suture: one-year outcomes. Retina. 2018;38(7):1377-1384. doi:10.1097/IAE.0000000000001692

17. Goel N. Clinical outcomes of combined pars plana vitrectomy and trans-scleral 4-point suture fixation of a foldable intraocular lens. Eye. 2018;32(6):1055-1061. doi:10.1038/s41433-018-0018-2

18. Patel NA, Shah P, Yannuzzi NA, et al. Clinical outcomes of 4-point scleral fixated 1-piece hydrophobic acrylic equiconvex intraocular lens using polytetrafluoroethylene suture. Clin Ophthalmol. 2018;12:2145-2148. doi:10.2147/OPTH.S174211

19. Khan MA, Gupta OP, Pendi K, et al. PARS plana vitrectomy with anterior chamber versus gore-tex sutured posterior chamber intraocular lens placement: long-term outcomes. Retina. 2019;39 (5):860-866. doi:10.1097/IAE.0000000000002042
20. Ohr MP, Wisely CE. Refractive outcomes and accuracy of IOL power calculation with the SRK/T formula for sutured, scleral-fixated Akreos AO60 intraocular lenses. Graefe's Arch Clin Exp Ophthalmol. 2020;258(10):2125-2129. doi:10.1007/s00417-02004721-9

21. Heuer DK, Barton K, Grehn F, Shaarawy T, Sherwood M Consensus on definitions of success. Guidel Des Report Glaucoma Surg Trials; 2009:15-24. Available from: http://www.icoph.org/dynamic/attach ments/resources/guidelinesglaucomasurgicaltrials.pdf. Accessed February 16, 2021.

22. Fontenot JL, Bona MD, Kaleem MA, et al. Vision rehabilitation preferred practice Pattern ${ }^{\circledR}$. Ophthalmology. 2018;125(1):P228P278. doi:10.1016/j.ophtha.2017.09.030

23. Su D, Stephens JD, Obeid A, et al. Refractive outcomes after pars plana vitrectomy and scleral fixated intraocular lens with gore-tex suture. Ophthalmol Retin. 2019;3(7):548-552. doi:10.1016/j. oret.2019.02.012

24. Botsford BW, Williams AM, Conner IP, Martel JN, Eller AW. Scleral fixation of intraocular lenses with gore-tex suture: refractive outcomes and comparison of lens power formulas. Ophthalmol Retin. 2019;3(6):468-472. doi:10.1016/j.oret.2019.02.005

25. Day HR, Durrani AK, Kim SJ, Patel S. Outcomes and complications of concurrent pars plana vitrectomy and scleral-fixated intraocular lens placement using gore-tex suture. J Vitreoretin Dis. 2020;4 (2):119-124. doi:10.1177/2474126419895691

26. Frisina R, Pinackatt SJ, Sartore M, et al. Cystoid macular edema after pars plana vitrectomy for idiopathic epiretinal membrane. Graefe's Arch Clin Exp Ophthalmol. 2014;253(1):47-56. doi:10.1007/s00417014-2655-x

27. Eagling EM. Ocular damage after blunt trauma to the eye. Its relationship to the nature of the injury. $B r J$ Ophthalmol. 1974;58 (2):126-140. doi:10.1136/bjo.58.2.126

28. Werner L, Wilbanks G, Nieuwendaal CP, et al. Localized opacification of hydrophilic acrylic intraocular lenses after procedures using intracameral injection of air or gas. J Cataract Refract Surg. 2015;41 (1):199-207. doi:10.1016/j.jcrs.2014.10.025

29. Patel NA, Fan KC, Yannuzzi NA, et al. Akreos AO60 intraocular lens opacification after retinal detachment repair. Ophthalmol Retin. 2020;4(8):854-856. doi:10.1016/j.oret.2020.03.030

30. Heiner P, Ligabue E, Fan A, Lam D. Safety and effectiveness of a single-piece hydrophobic acrylic intraocular lens (enVista ${ }^{\circledR}$ ) Results of a European and Asian-Pacific study. Clin Ophthalmol. 2014;8:629-635. doi:10.2147/OPTH.S56135

31. Manoharan N, Prasad P. Secondary Intraocular Lens. Intech; 2019. doi:10.5772/intechopen.89569

32. Abd-elhafez YA, Ahmed A, Ghali A, et al. Evaluation of flanged haptics intrascleral sutureless intraocular lens fixation. Egyptian J Hosp Med. 2019;76:3718-3723.
Clinical Ophthalmology

\section{Publish your work in this journal}

Clinical Ophthalmology is an international, peer-reviewed journal covering all subspecialties within ophthalmology. Key topics include: Optometry; Visual science; Pharmacology and drug therapy in eye diseases; Basic Sciences; Primary and Secondary eye care; Patient Safety and Quality of Care Improvements. This journal is indexed on PubMed
Central and CAS, and is the official journal of The Society of Clinical Ophthalmology (SCO). The manuscript management system is completely online and includes a very quick and fair peer-review system, which is all easy to use. Visit http://www.dovepress.com/ testimonials.php to read real quotes from published authors.

\section{Dovepress}

